\title{
Rural Logistics Demand Forecast Based on Gray Neural Network Model
}

\author{
Zhengyi Yang \\ Guilin University of Aerospace Technology, Guilin Guangxi, 541004, China
}

Keywords: Gray neural network model, Rural logistics, Demand forecast.

\begin{abstract}
As an important part of rural economy, rural logistics plays an important role in people's daily life. In order to reflect the changing rule of rural logistics demand more accurately and comprehensively, it is necessary to introduce a scientific prediction model, that is, a gray neural network model. This paper analyzes the demand forecast of rural logistics based on gray neural network model and starts from the status quo of the basic demand of rural logistics. Then it discusses the principle of rural logistics demand forecast and the rural logistics demand forecast of the combined model, and then the demand of rural logistics based on gray neural network model is also simulated. Finally, the significance of rural logistics demand forecast based on gray neural network model is discussed.
\end{abstract}

\section{Introduction}

In recent years, with the rapid economic development of our country, people also pay more attention to the logistics industry. Coupled with the increasingly fierce market competition and the advent of economic globalization, China's logistics volume has shown a substantial increase. Especially in recent years, the demand for rural logistics has been gradually expanded, which has greatly improved the rural economy. It is precisely because of this that rural logistics has become a new way to develop the rural economy. This makes the relevant departments of the state and government attach great importance to the demand of rural logistics. In order to promote the rapid development of rural logistics and expand the rural logistics demand further, the scholars studied and put forward the gray neural network model prediction method. The principle of this forecasting method is based on the complex relationship between the size of rural logistics demand and many factors. The purpose of model establishment is to reflect the complexity of rural logistics demand scientifically and comprehensively, and to improve the prediction accuracy of rural logistics demand, and to grasp the change rule of rural logistics demand prediction finally.

\section{The status quo of basic needs of rural logistics}

The concept of rural logistics is not the same as the agricultural logistics, agricultural products logistics, circulation of agricultural products as we often say, but they all contain others or are contained, rural logistics just highlights the dispersiveness, seasonality, differences , diversity and other characteristics of rural logistics. The following author will lead you to understand the current status of the basic needs of rural logistics.

From the perspective of the rural economy as a whole, the distribution of agricultural production in our country is extremely unbalanced. There are not only great differences in product quality and production technology, but also in the areas of high yielding areas or harvest years, there is a phenomenon of increasing output without increasing income. Of course, the biggest constraint at present is not the market factor but the agricultural product logistics.

For the development of rural economy, the production of agricultural products is a crucial link. However, once the agricultural products are produced, but can not be sold, it will directly lead to low prices and hurt agriculture. This kind of production disadvantage gradually began to flow from the production area to the circulation area. It also made the rural logistics industry an important measure to improve the structure of the rural economy and also the key way to deal with the conflicts between the big market and small-scale production. At this stage, China's main form of rural logistics mainly is 
the agricultural product logistics, while the main form of the agricultural product logistics is mainly the normal-temperature logistics and the natural logistics, which makes many agricultural products great decay or impact in the process of logistics and cause transportation loss.

In recent years, in particular, news reports have reported that many merchants in rural areas in China can not transport vegetables such as vegetables or fruits they produce and can only let them rot in the fields. The reason for these problems is that the logistics gap of agricultural products in our country is too large to meet the requirements of external transportation of agricultural products. In addition, some traders in close quarters brought a large amount of agricultural products together, resulting in oversupply of agricultural products and greatly reducing the market prices of these agricultural products. With the rapid economic development of our country, people's living standards have also been greatly improved. Naturally, people are not only seeking food and clothing, but higher levels of material and spiritual enjoyment. For example, they pay more attention to the safety, hygiene and freshness of agricultural products, etc. However, taking into account that most of the distribution of agricultural products in China is relatively dispersed, therefore, higher requirements have been put forward in terms of processing technology , logistics and transportation of agricultural product .

It is undeniable that the logistics facilities in most rural areas in our country are still not perfect, which can not meet the standards and requirements of modernization. In the whole process of production, processing and transportation of agricultural products, the shortage of logistics and transportation lead to problems in the whole set of agricultural products, which hinder the real logistics, funds flow and information flow in rural areas. Therefore, if we want to vigorously develop the rural economy, we must perfect the product service, information services and other social services of rural logistics. The foundation and premise for these services to be realized is to establish a modern rural logistics system.

To sum up, rural logistics has become a new bottleneck in the current rural economic construction and development. We must find a scientific and effective forecasting model and make a precise and reliable forecast of rural logistics needs.

\section{The principle of the rural logistics demand forecast}

Rural logistics, as the more advanced forms of rural economic organizations and management tools, has become the third source of profits for reducing the material consumption and increasing labor productivity in rural areas. It can be said that rural logistics has become an important backbone of rural economic development. The so-called demand for rural logistics refers to the general term for transportation, handling, loading and unloading, processing, storage and other activities that occur in all production, living and other economic activities in rural areas. However, the factors that directly affect the demand of rural logistics are the changes of the rural economic structure, the individualization and diversification of consumer demand.

As for the forecasting principle of rural logistics demand, we need to combine with the development status of rural logistics market and the factors related to the fluctuation of rural logistics market, and then we use scientific and effective logistics technology methods, experience judgment and prediction to realize the analysis and judgment of rural logistics demand. Finally, according to the predictions obtained, the changing rules and trends of the rural economic markets are analyzed and summarized, and the strategies and methods that can improve the level of rural economic development can be drawn. The mathematical model of rural logistics demand can be expressed as:

$\mathrm{Y}=\mathrm{f}(\mathrm{x} 1, \mathrm{x} 2, \ldots, \mathrm{xn})$, in this mathematical formula, $Y$ refers to the logistics demand, $\mathrm{x} 1$ refers to the factors that affect the logistics needs, including economic factors, social factors and environmental factors. The basic logistics demand forecast process is to obtain the logistics demand historical data firstly, then forecast the data, and then start to select the logistics forecast model to adjust the data, and finally to forecast the rural logistics demand. Therefore, the selection of a scientific and correct prediction model has a great significance and role for the accuracy and reliability of the rural logistics demand forecast. Based on the fact that the current demand of rural logistics is affected by many factors of social activities and the nonlinear relationship between these factors appears, the 
requirements for the prediction accuracy of the model will be higher and more stringent. Therefore, this paper selects the gray neural network model, and explore the rural logistics demand forecast situation based on the forecast model.

\section{Rural logistics demand forecast of the combined model}

The gray neural network model is described in this paper essentially refers to the combination of the gray prediction model and the BP neural network prediction model, which is also the inevitable choice under the ever-changing and complex factors of the current logistics demand. The following author discusses and analyzes the two models, thereby deepens the understanding of readers on the gray neural network prediction model.

\subsection{Gray prediction model}

The so-called gray prediction is essentially the use of gray model to establish a mathematical model of the target prediction system. In general, in order to eliminate the trend,the modeling is the use of differential processing on selected data by the way of Time series analysis. However, gray modeling is different from the modeling approach is to accumulate the original data by the first, and then carried out an arrangement to new data series with form regular. Then the differential equation is established, the future development state of the target system is analyzed from the equation, and finally the prediction is made. The following is an analysis of the equation constructed by the gray prediction model.

GM (m, n) , this formula expresses a gray model of m-order and n-variables, but in practice, the gray dynamic model of GM $(1,1)$ is generally used, which can also be called a first order differential equation with only one variable. Specific application to the forecast of rural logistics needs, it can be described as $\frac{\mathrm{dx}(1)}{\mathrm{dt}}+\alpha \mathrm{x}(1)=\mathrm{u}$, And in this equation, $\mathrm{u}$ represents the estimated parameter in the differential agenda. Next, the parameter to be evaluated $\alpha$ and $\mathrm{u}$ can be solved by $\mathrm{x}(1)(\mathrm{i}+1)=\left(\mathrm{x}(1)-\frac{\mathrm{u}}{\alpha}\right) \mathrm{e}^{-\infty}+\frac{\mathrm{u}}{\alpha}$. Finally, the derived values of $\alpha$ and $\mathrm{u}$ are substituted into the first-order differential equations to obtain the GM $(1,1)$ prediction model.

\subsection{BP neural network prediction model}

The so-called BP neural network prediction model is also called error back propagation neural network, which is different from the gray prediction model, and it is a multi-layer forward network with remarkable unidirectionality. At the same time, BP neural network is also one of the most widely used prediction models. Due to its simple structure and easy operation, it can display nonlinear input and output relations arbitrarily. It is easy to show obvious advantages on nonlinear approximation ability and generalization ability, fitness and other aspects, which are very favorable for rural logistics demand forecast. Its function formula can be expressed as $f(x)=\frac{1}{1+e-x}$.

\subsection{The combination of rural logistics demand forecast model - gray neural network prediction model}

Compared with the former two prediction models, the gray neural network prediction model integrates the advantages of the gray prediction model and the BP neural network prediction model, and it is widely applied in the field of logistics such as rural logistics because of its simple calculation and high prediction accuracy. In particular, the gray neural network prediction model can not only make up for the shortcomings that the gray prediction model can not solve complex nonlinear functions, but also clearly reflects the changing rules and trends of input and output of the logistics data. The application process of the whole gray neural network prediction model in the rural logistics demand is roughly to use BP neural network to organize and train the historical data of rural logistics in order to integrate the input and output of data and get the best BP neural network right value. Then 
the gray prediction model is used to predict the future input value of the neural network, and the forecast value of the model is used as the input value of the BP neural network to forecast the rural logistics demand.

\section{Simulation research based on gray neural network model of rural logistics demand forecast}

\subsection{Data source of rural logistics}

The simulation data of logistics from the Statistical Yearbook of Hunan Province, and respectively, with the parameters $\mathrm{x} 1, \mathrm{x} 2, \mathrm{x} 3, \mathrm{x} 4, \mathrm{x} 5, \mathrm{x} 6, \mathrm{x} 7, \mathrm{x} 8$ on behalf of the rural GDP, primary industry in rural areas, the total secondary industry , Gross value of tertiary industry, total amount of rural foreign trade, total retail sales of rural consumer goods, consumption level of rural residents and total volume of rural logistics. And with $x$ on behalf of the logistics input, $y$ on behalf of the logistics output.

\subsection{The normalization processing of rural logistics data}

Predicting rural logistics demand based on BP neural network model may affect the forecast accuracy of rural logistics demand due to the speed of neural network learning. Therefore, to enhance the learning speed of neural network must be pre-processing of rural logistics data information, which is the so-called normalization. The basic formula is as follows: $\mathrm{T}=\frac{X-X \min }{X \max -\mathrm{xmin}} * 0.6+0.1$, where Xmax represents the maximum of the original rural logistics data, Xmin represents the minimum value of rural logistics raw data, $\mathrm{X}$ represents the original data, T represents the processed Of the data, that is normalized data. After the completion of the forecast of rural logistics demand, the logistics data of the anti-normalized formula is: $X=\mathrm{xmin}+\frac{(T-0.1)(X \max -X \min )}{0.6}$

\section{The Significance of Rural Logistics Demand Forecast Based on Gray Neural Network Model}

Demand forecast of rural logistics based on gray neural network model is an important measure to further develop rural logistics. The success of this measure will play an important role in rural economic development. The following author makes a simple analysis of the significance of the success of the rural logistics renewal forecasting based on the gray neural network model to the rural economic development.

First of all, it is good for agricultural production and circulation. The sales and circulation of agricultural products need to be promoted and coordinated, and thus the blindness of agricultural production and circulation can be reduced so as to form a special production and distribution channel to ensure the quality and speed of product transportation.

Second, farmers can get employed by increasing opportunities. As a large number of migrant workers go to the city to work, making rural unemployment more and more serious. Therefore, the development of rural logistics is conducive to the formation of a new social division of labor, increase employment opportunities for migrant workers, which can ensure the stability and sustainability of rural economic development.

Finally, the income of farmers needs to be increased. Rural logistics has now become an important part of rural economic development, and an excellent rural logistics service not only provides consumers with excellent service, but also enhances the income of farmers. Needless to say, the development of rural logistics has increased the income of peasants and indirectly also improved the standard of living of peasants.

\section{Conclusion}

In summary, we can basically understand that the logistics industry is one of the area that able to reflect the economic development status and level of the field most, and the prosperity of rural economy behind the rapid development of rural logistics. However, the development of rural 
economy also makes the demand forecast of rural logistics become more and more complex, apparently this is very unfavorable to the development and progress of rural logistics. The gray neural network model is the best forecasting method for this problem. Its perfect integration with BP neural network algorithm improves the accuracy of rural logistics demand forecast, and also reflects the changing rule of rural logistics demand more comprehensively and carefully. It can be said that the application of gray neural network model in rural logistics demand forecast has brought very obvious forecasting effect and has great development prospects.

\section{References}

[1] Li Xiaoli, Wang Zejiang, coal logistics demand forecast model based on improved gray neural network, Statistics and Decision-Making, 2015-09-29.

[2] Wang Zejiang, coal logistics demand forecast based on gray neural network, Taiyuan University of Technology, 2014-05-01.

[3] Wang Yingying, coal logistics demand forecast based on gray neural network model, Beijing Jiaotong University, 2012-06-01.

[4] Yan Juan, gray neural network model in the logistics demand forecast, Computer Simulation, 2011-07-15.

[5] Yu Da, Zhejiang road logistics demand forecast based on gray neural network, Zhejiang University of Technology, 2009-12-01. 